\title{
Representation of Solid, Liquid, and Vapor phases of Binary Lennard Jones Mixtures using the SLV-EoS
}

\author{
P. Stringari ${ }^{1}$, M. Campestrini ${ }^{1}$, C. Coquelet $^{1}$, and P. Arpentinier ${ }^{2}$ \\ ${ }^{1}$ Centre Thermodynamique des Procédés (CTP), Mines ParisTech, Fontainebleau 77305, France \\ ${ }^{2}$ Air Liquide - Centre de Recherche Claude-Delorme, Jouy-en-Josas 78354, France
}

\begin{abstract}
The capability of the SLV-EoS, recently proposed by A. Yokozeki [1], of representing the phase diagrams for binary mixtures of Lennard-Jones $(\mathrm{LJ})$ fluids has been investigated in this work. The SLV-EoS has been used for producing the phase diagrams of binary Lennard-Jones mixtures with diameter ratio $\sigma_{11} / \sigma_{22}$ ranging from 0.85 and 0.95 , and well-depth ratio $\varepsilon_{11} / \varepsilon_{22}$ ranging from 0.625 and 1.6 at reduced pressure $P^{*}=P \sigma_{11}{ }^{3} / \varepsilon_{11}=0.002$. The obtained phase diagrams have been compared with the corresponding results obtained by Monte-Carlo (MC) simulation [2-5].
\end{abstract}

\section{Introduction}

Solid-liquid phase diagrams are usually classified in six types: solid solution, azeotrope, eutectic with partial immiscibility, eutectic with complete immiscibility, peritectic with eutectic, and molecular compound [6], see Fig. 1. "Classical" modeling approach for calculating SLE, as reported for instance by Gmehling and Kolbe [7], assumes solid phases as pure compounds. This assumption is of practical utility in representing types of phase diagrams with total immiscibility in the solid phases. Nevertheless, this approach cannot be used for systems presenting partial or total miscibility in the solid phase. In [1] Yokozeki proposed a non-cubic analytical EoS, named SLV-EoS, for describing the phase behavior of three thermodynamic states (solid, liquid, and vapor) of matter. In this work, the authors tested the capability of the SLV-EoS in representing the SLE of systems with partial or total miscibility in the solid phase.
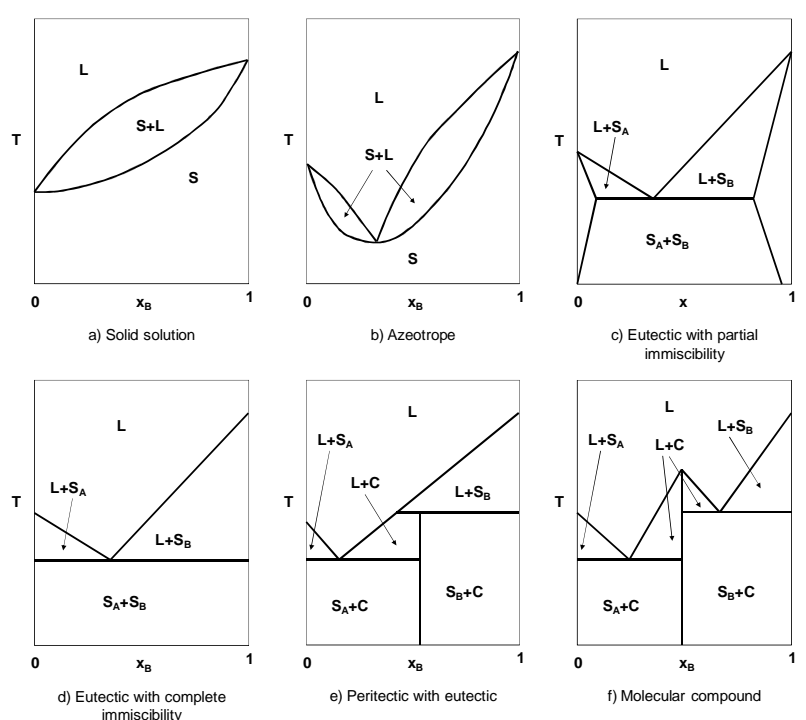

Figure 1. Types of solid-liquid phase diagrams identified by Matsuoka [1]: (a) solid solution, (b) azeotrope, (c) eutectic with partial immiscibility, (d) eutectic with complete immiscibility,

(e) peritectic with eutectic, and (f) molecular compound.

The study has been carried out for LJ fluids, considered as very good approximations of real fluids constituted of "simple" molecules. Our results were compared with phase diagrams including solid phases for binary mixtures of LJ components studied in References 2-5 using MC simulation.

\section{SLV-EoS for pure LJ fluids}

In order to represent the molecular simulation results for LJ fluids, SLV-EoS [1] has been written in terms of reduced variables: 
$P^{*}=\frac{T^{*}}{v^{*}-b^{*}} \frac{v^{*}-d^{*}}{v^{*}-c^{*}}-\frac{a^{*}}{v^{* 2}}$

Where:

$$
\begin{aligned}
& P^{*}=\frac{P \sigma^{3}}{\varepsilon} ; \quad T^{*}=\frac{k_{B} T}{\varepsilon} ; \quad v^{*}=\frac{v}{N_{A} \sigma^{3}} \\
& a^{*}=\frac{a}{N_{A}^{2} \varepsilon \sigma^{3}} ; \quad b^{*}=\frac{b}{N_{A} \sigma^{3}} ; \\
& c^{*}=\frac{c}{N_{A} \sigma^{3}} ; \quad d^{*}=\frac{d}{N_{A} \sigma^{3}}
\end{aligned}
$$

The SLV-EoS [1] can easily be obtained from Eq. (1) substituting the expressions for the reduced variables, eq. (2), and considering $R=N_{A} k_{B} \cdot N_{A}$ is the Avogadro number, and $k_{B}$ is Boltzmann constant. Parameters $a^{*}$ and $b^{*}$ are functions of temperature, as in the original SLV-EoS:

$$
\begin{aligned}
& a^{*}\left(T^{*}\right)=a_{0}+a_{1} T^{*} \exp \left(-a_{2} T^{*_{n}}\right) \\
& b^{*}\left(T^{*}\right)=b_{0}+b_{1} \exp \left(-b_{2} T^{* m}\right)
\end{aligned}
$$

Parameters of the SLV-EoS in reduced variables, Eq. (1), have been regressed for reproducing analytically the temperatures and pressures of the critical and triple point for a pure LJ fluid. Temperature and pressure values at the critical point were taken from [8]: $T_{c}^{*}=1.31$ and $P_{c}^{*}=0.126$. Temperature and pressure values at the triple point were taken from [9]: $T_{t}^{*}=0.692$ and $P_{t}^{*}=1.21 \times 10^{-3}$. The temperature dependence in parameters $a^{*}$ and $b^{*}$ of eq. (1) have been regressed in order to represent saturation, melting, and sublimation reduced pressures for a given reduced temperature. Reduced pressure for vapor-liquid equilibrium data of pure LJ fluids were taken from Reference 8, while reduced pressure for solid-liquid and solid-gas were taken from Reference 9. Parameters of eq. (1) are presented in Tab. 1. A $P^{*}-T^{*}$ graphical comparison between molecular simulation results and the LJ SLV-EoS, eq. (1), is presented in Fig. 2 for the pure LJ fluid.
Table 1. Parameters of the SLV-EoS in reduced variables, eqs (1-4).

\begin{tabular}{ccc}
\hline Parameter & Value & Units \\
\hline$a_{0}$ & 4.44121 & - \\
$a_{1}$ & $1.36198 \times 10^{3}$ & - \\
$a_{2}$ & 6.95289 & - \\
$n$ & 0.334556 & - \\
$b_{0}$ & 1.38704 & - \\
$b_{1}$ & -1.56221 & - \\
$b_{2}$ & 0.45189 & - \\
$m^{*}$ & 2.76444 & - \\
$c^{*}$ & 1.43937 & - \\
$d^{*}$ & 1.42892 & - \\
$N_{\mathrm{A}}$ & $6.022141793 \times 10^{23}$ & $\mathrm{~mol}^{-1}$ \\
$k_{\mathrm{B}}$ & $1.380648813 \times 10^{-23}$ & $\mathrm{~J} / \mathrm{K}$ \\
\hline
\end{tabular}

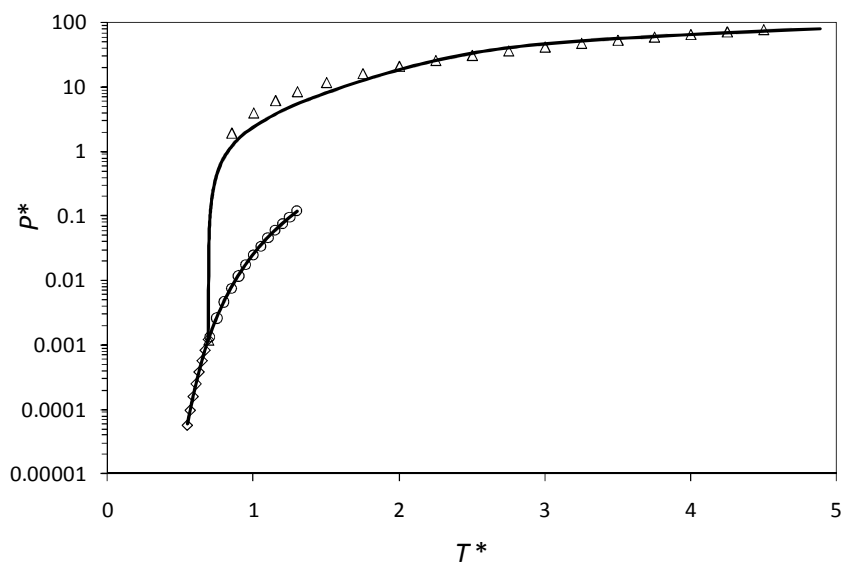

Figure 2. Reduced pressure, $P^{*}$, versus reduced temperature, $T^{*}$, diagram for a pure LJ fluid. Symbols represent molecular simulation results.

०: VLE [8]; $\square$ SVE [9]; $\Delta$ : SLE [9];

-: LJ SLV-EoS, eq. (1).

\section{SLV-EoS for mixtures}

The following mixing rules were used for $a^{*}, b^{*}, c^{*}$, and $d^{*}$ :

$$
\begin{aligned}
& a^{*}=\sum_{i=1}^{N} \sum_{J=1}^{N} \sqrt{a_{i}^{*} a_{j}^{*}}\left(1-k_{i j}\right) x_{i} x_{j}, \\
& b^{*}=\frac{1}{2} \sum_{i, j=1}^{N}\left(b_{i}^{*}+b_{j}^{*}\right)\left(1-m_{i j}\right) x_{i} x_{j}, \\
& c^{*}=\frac{1}{2} \sum_{i, j=1}^{N}\left(c_{i}^{*}+c_{j}^{*}\right)\left(1-n_{i j}\right) x_{i} x_{j}, \\
& d^{*}=\frac{1}{2} \sum_{i, j=1}^{N}\left(d_{i}^{*}+d_{j}^{*}\right)\left(1-l_{i j}\right) x_{i} x_{j},
\end{aligned}
$$

where $k_{i j}=k_{j i}, \quad m_{i j}=m_{j i}, l_{i j}=l_{j i}, n_{i j}=n_{j i}$ and $k_{i i}=m_{i i}=n_{i i}=l_{i i}=0$. 


\section{Phase diagrams of binary LJ mixtures}

In References 2 to 5, the effect of the well-depth ratio, $\varepsilon_{11} / \varepsilon_{22}$, and the diameter ratio, $\sigma_{11} / \sigma_{22}$, on the phase diagram of binary LJ mixtures was studied. Phase diagrams were produced using MC simulation for diameter ratios ranging from 0.85 to 0.95 , and well-depth ratios ranging from 0.625 to 1.6 for a reduced pressure $P^{*}$ $=0.002$. These ranges of $\varepsilon_{11} / \varepsilon_{22}$ and $\sigma_{11} / \sigma_{22}$ allow obtaining phase diagrams (a), (b), and (c) of figure 1 . In this work, the capability of the SLV-EoS for LJ fluids (LJ SLV-EoS), eq. (1), of reproducing the phase diagrams produced with MC simulation has been investigated. Obtained results are represented in figures 3 to 11 . For each couple $\varepsilon_{11} / \varepsilon_{22}$ and $\sigma_{11} / \sigma_{22}$, graphs (a) represent the diagram obtained via MC simulation [3]. The MC simulation values are not presented in the papers, and are no more available from the authors; for these reason, these diagrams have been produced reading data points in the graphs presented in references 2-5. Graphs (b) in Figs. 3-11 present the phase diagrams obtained with the LJ SLV-EoS with binary interaction parameters (BIPs) equal to 0 (see eqs. 5-8). Graphs (c) in Figs. 3-11 present the phase diagrams obtained with the LJ SLV-EoS with binary interaction parameters regressed for reproducing the phase diagrams obtained with MC simulation. Regressed parameters are presented in Tab. 2. In figures $3-11$, the reduced variables $P^{*}=P \sigma_{11}^{3} / \varepsilon_{11}$ and $T^{*}=k_{B} T / \mathcal{E}_{11}$ are used.
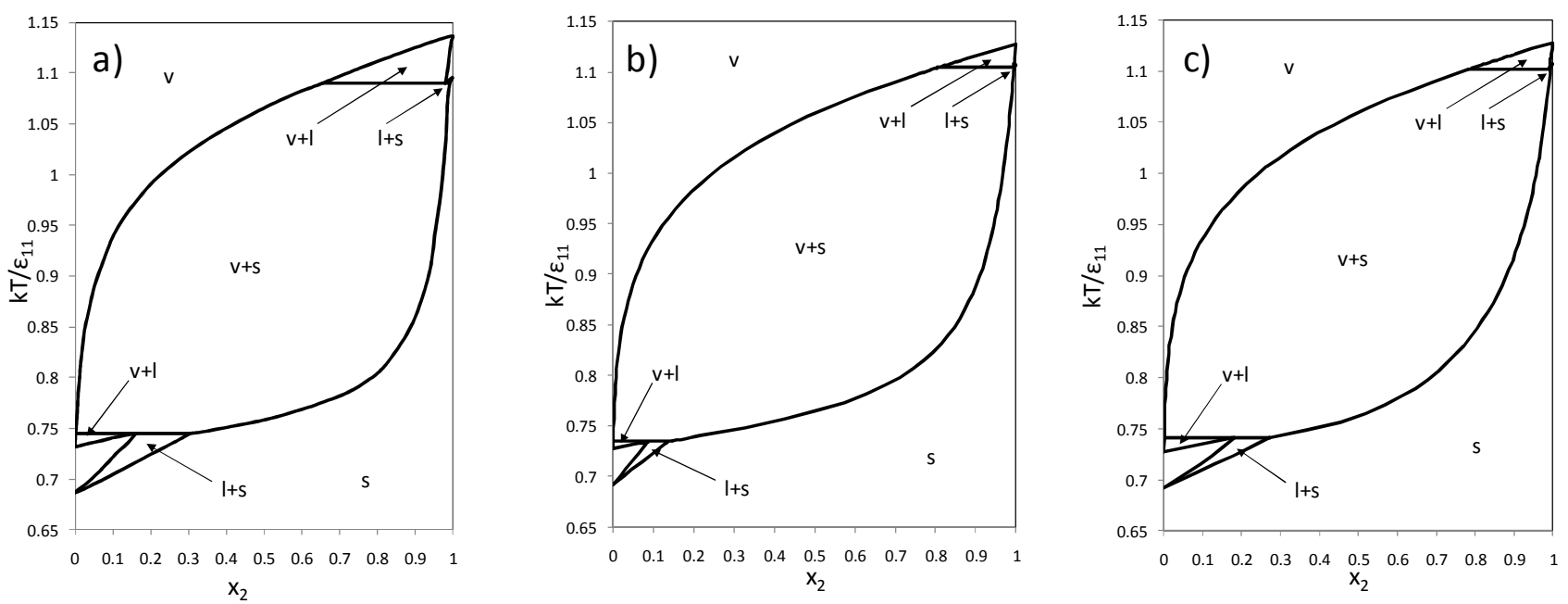

Figure 3. $T^{*}-\mathrm{x}_{2}$ phase diagrams for LJ binary mixtures with $\varepsilon_{11} / \varepsilon_{22}=0.625$ and $\sigma_{11} / \sigma_{22}=0.95$ at $P^{*}=0.002$.

(a) MC simulation diagram from [3]; (b) LJ SLV-EoS with null BIPs; (c) LJ SLV-EoS with regressed BIPs.
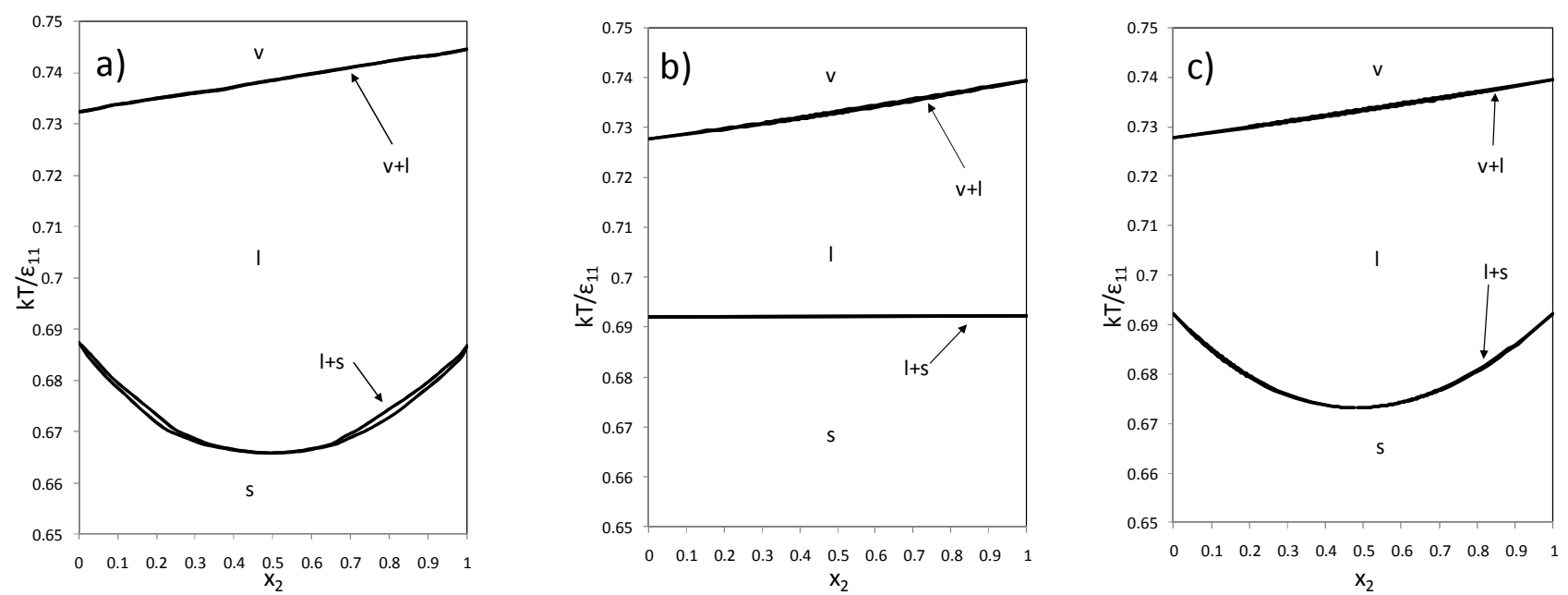

Figure 4. $T^{*}-\mathrm{x}_{2}$ phase diagrams for LJ binary mixtures with $\varepsilon_{11} / \varepsilon_{22}=1$ and $\sigma_{11} / \sigma_{22}=0.95$ at $P^{*}=0.002$.

(a) MC simulation diagram from [3]; (b) LJ SLV-EoS with null BIPs; (c) LJ SLV-EoS with regressed BIPs. 

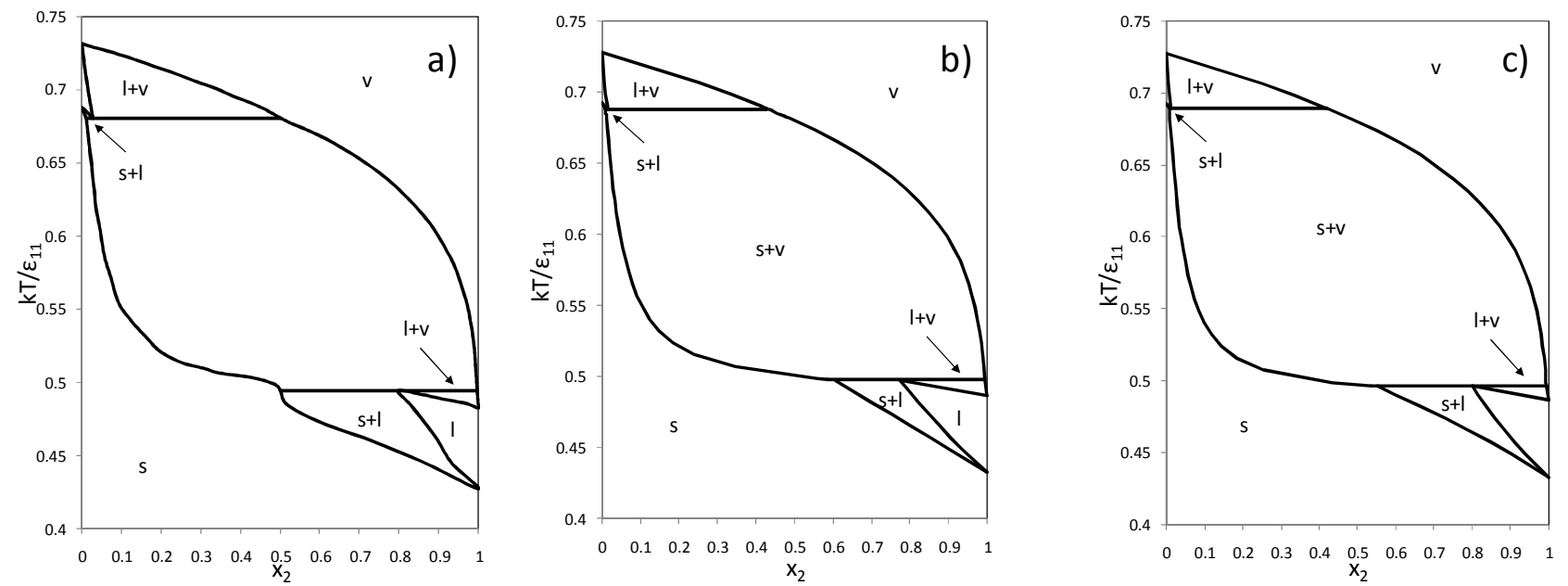

Figure 5. $T^{*}$ - $\mathrm{x}_{2}$ phase diagrams for LJ binary mixtures with $\varepsilon_{11} / \varepsilon_{22}=1.6$ and $\sigma_{11} / \sigma_{22}=0.95$ at $P^{*}=0.002$.

(a) MC simulation diagram from [3]; (b) LJ SLV-EoS with null BIPs; (c) LJ SLV-EoS with regressed BIPs.
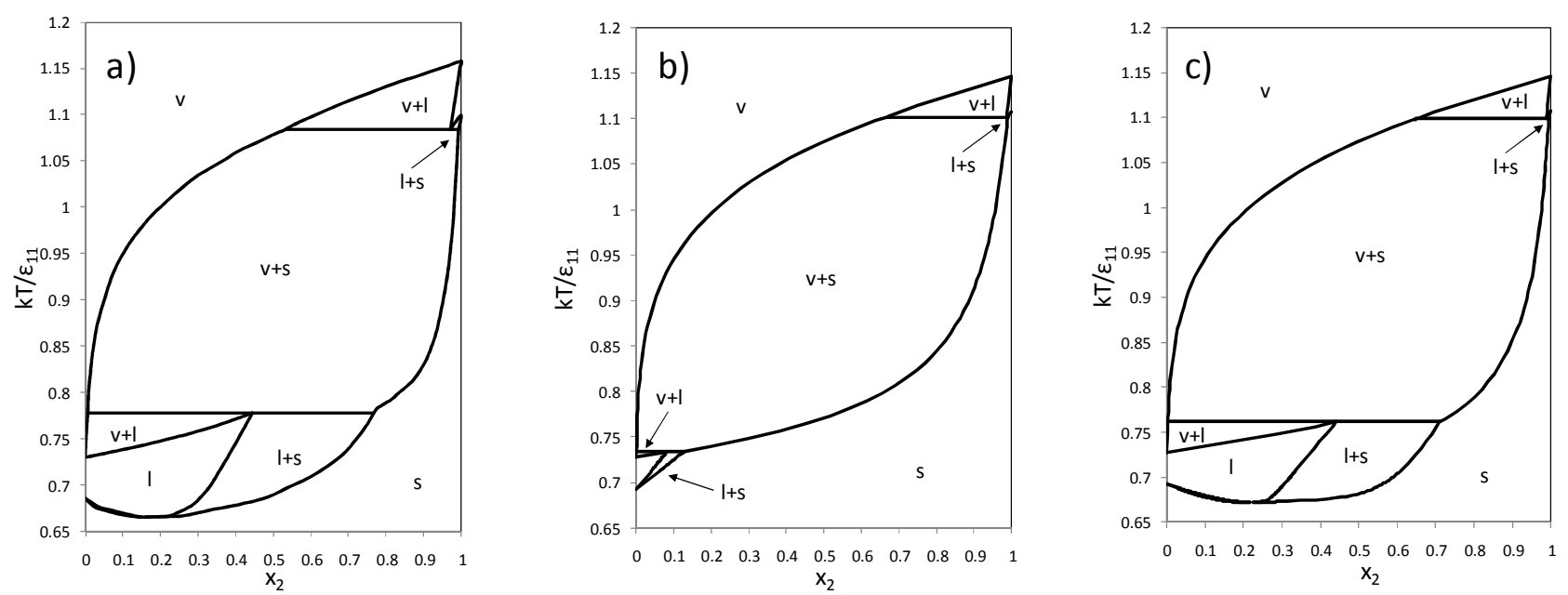

Figure 6. $T^{*}-\mathrm{x}_{2}$ phase diagrams for LJ binary mixtures with $\varepsilon_{11} / \varepsilon_{22}=0.625$ and $\sigma_{11} / \sigma_{22}=0.9$ at $P^{*}=0.002$. (a) MC simulation diagram from [3]; (b) LJ SLV-EoS with null BIPs; (c) LJ SLV-EoS with regressed BIPs.
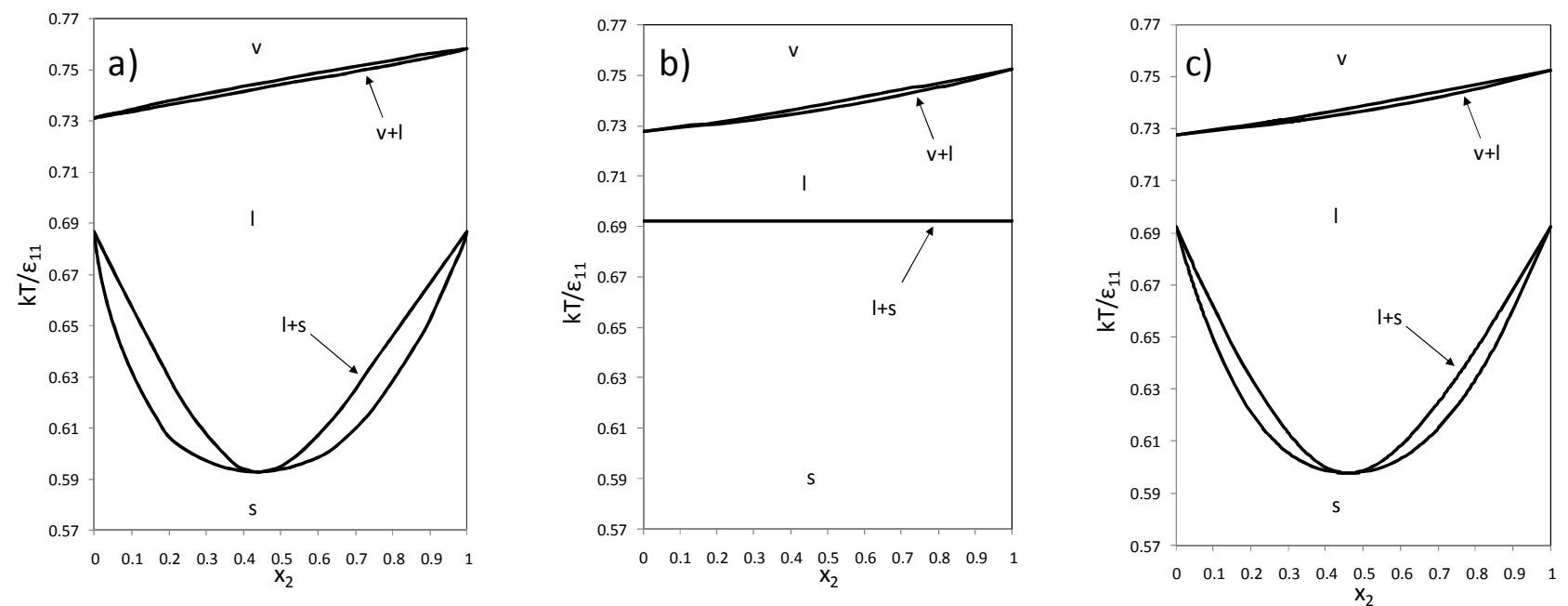

Figure 7. $T^{*}-\mathrm{x}_{2}$ phase diagrams for LJ binary mixtures with $\varepsilon_{11} / \varepsilon_{22}=1$ and $\sigma_{11} / \sigma_{22}=0.9$ at $P^{*}=0.002$. (a) MC simulation diagram from [3]; (b) LJ SLV-EoS with null BIPs; (c) LJ SLV-EoS with regressed BIPs. 

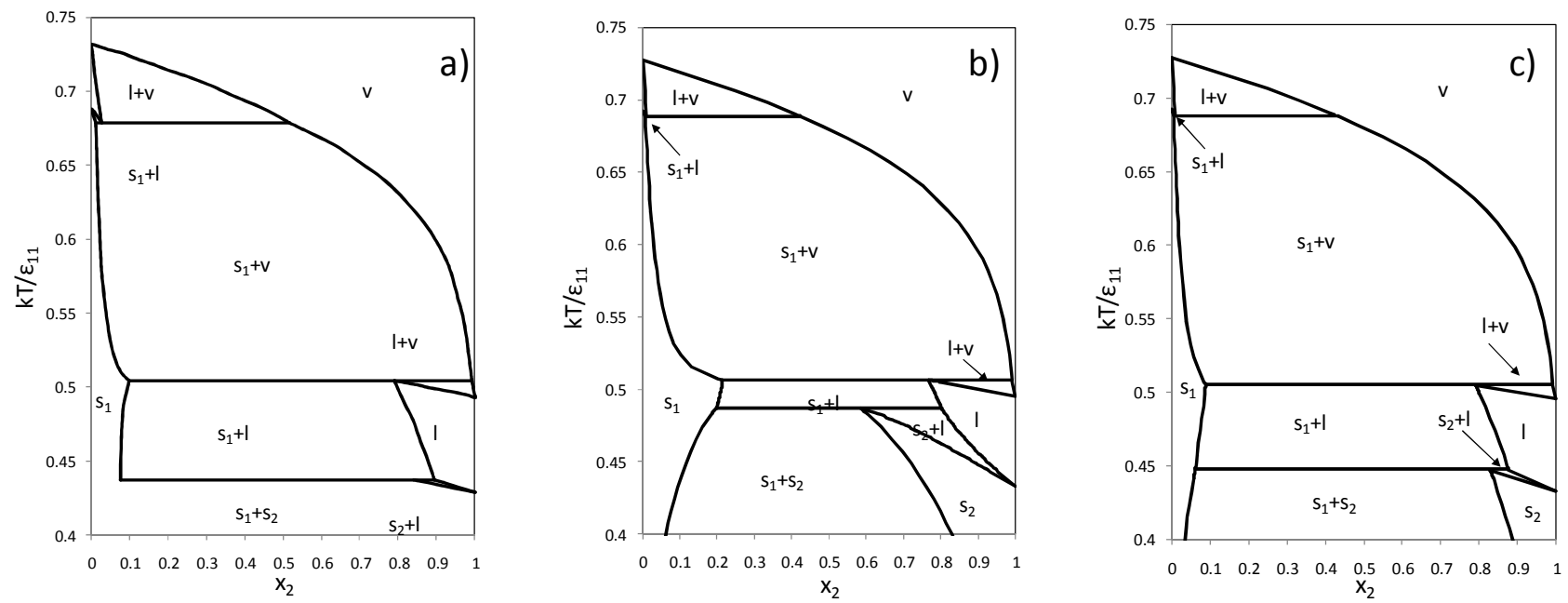

Figure 8. $T^{*}-\mathrm{x}_{2}$ phase diagrams for LJ binary mixtures with $\varepsilon_{11} / \varepsilon_{22}=1.6$ and $\sigma_{11} / \sigma_{22}=0.9$ at $P^{*}=0.002$.

(a) MC simulation diagram from [3]; (b) LJ SLV-EoS with null BIPs; (c) LJ SLV-EoS with regressed BIPs.
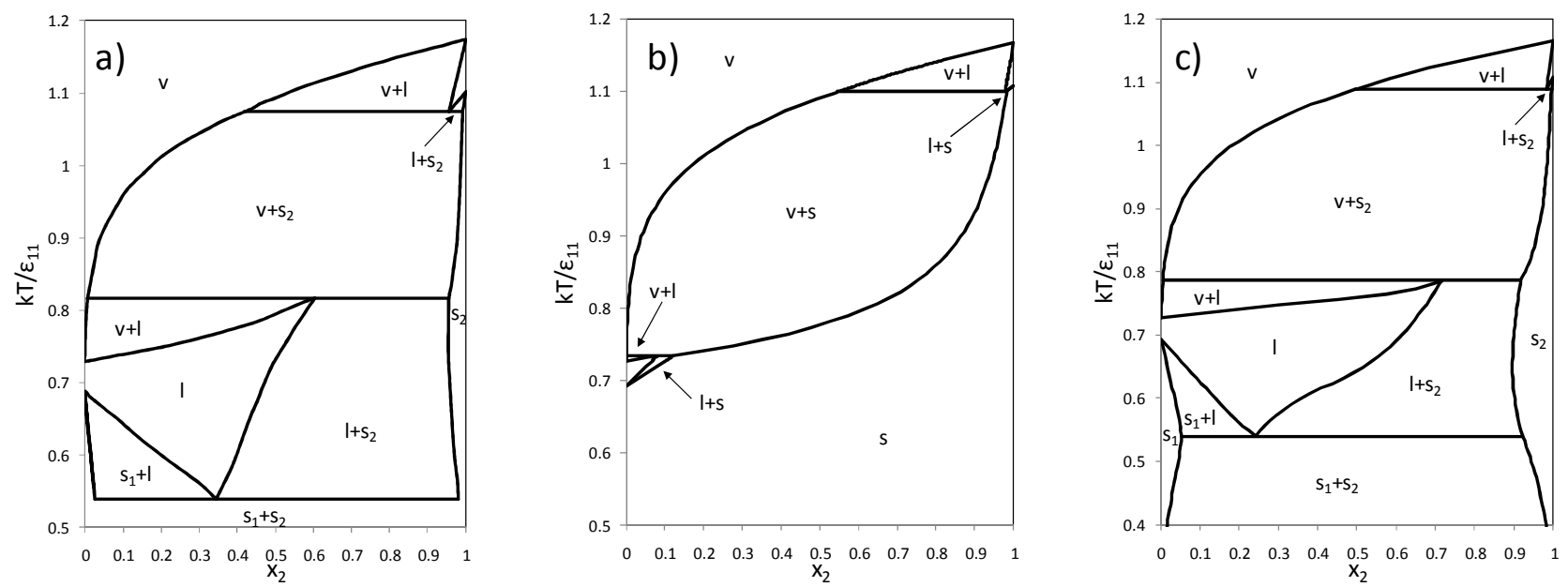

Figure 9. $T^{*}$ - $\mathrm{x}_{2}$ phase diagrams for LJ binary mixtures with $\varepsilon_{11} / \varepsilon_{22}=0.625$ and $\sigma_{11} / \sigma_{22}=0.85$ at $P^{*}=0.002$.

(a) MC simulation diagram from [3]; (b) LJ SLV-EoS with null BIPs; (c) LJ SLV-EoS with regressed BIPs.
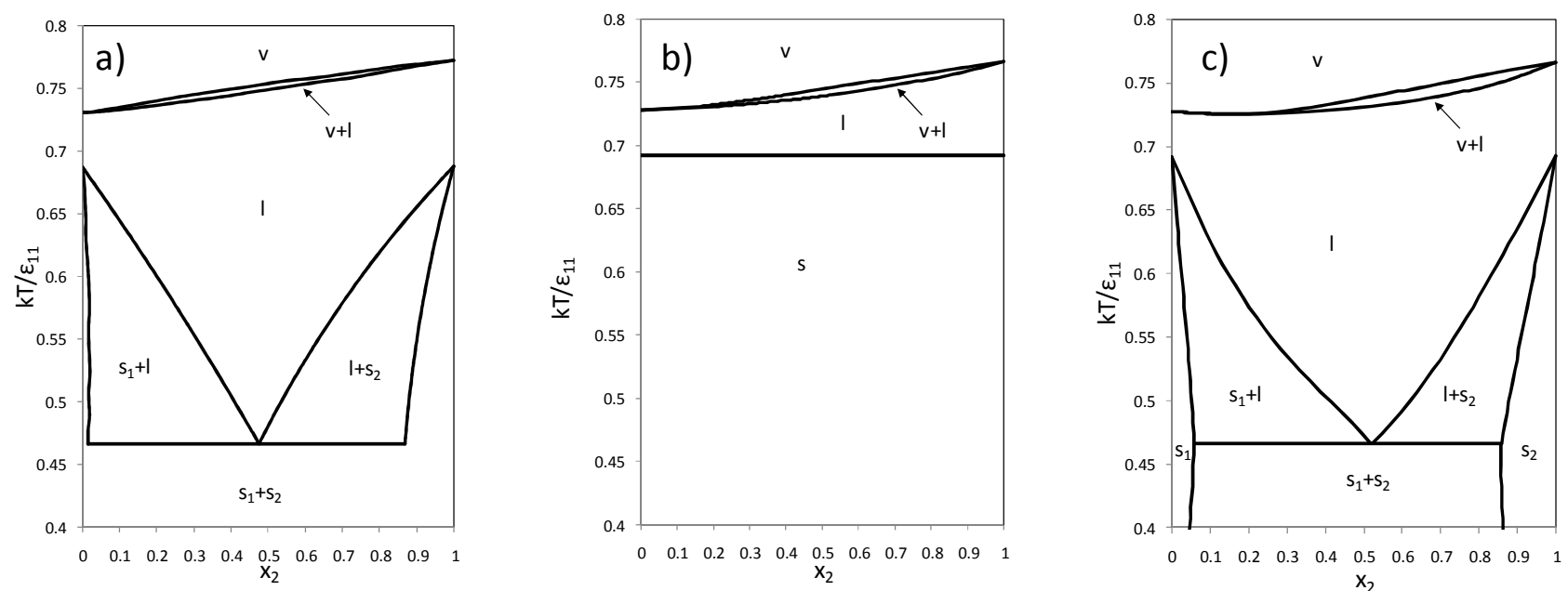

Figure 10. $T^{*}-\mathrm{x}_{2}$ phase diagrams for LJ binary mixtures with $\varepsilon_{11} / \varepsilon_{22}=1$ and $\sigma_{11} / \sigma_{22}=0.85$ at $P^{*}=0.002$.

(a) MC simulation diagram from [3]; (b) LJ SLV-EoS with null BIPs; (c) LJ SLV-EoS with regressed BIPs. 

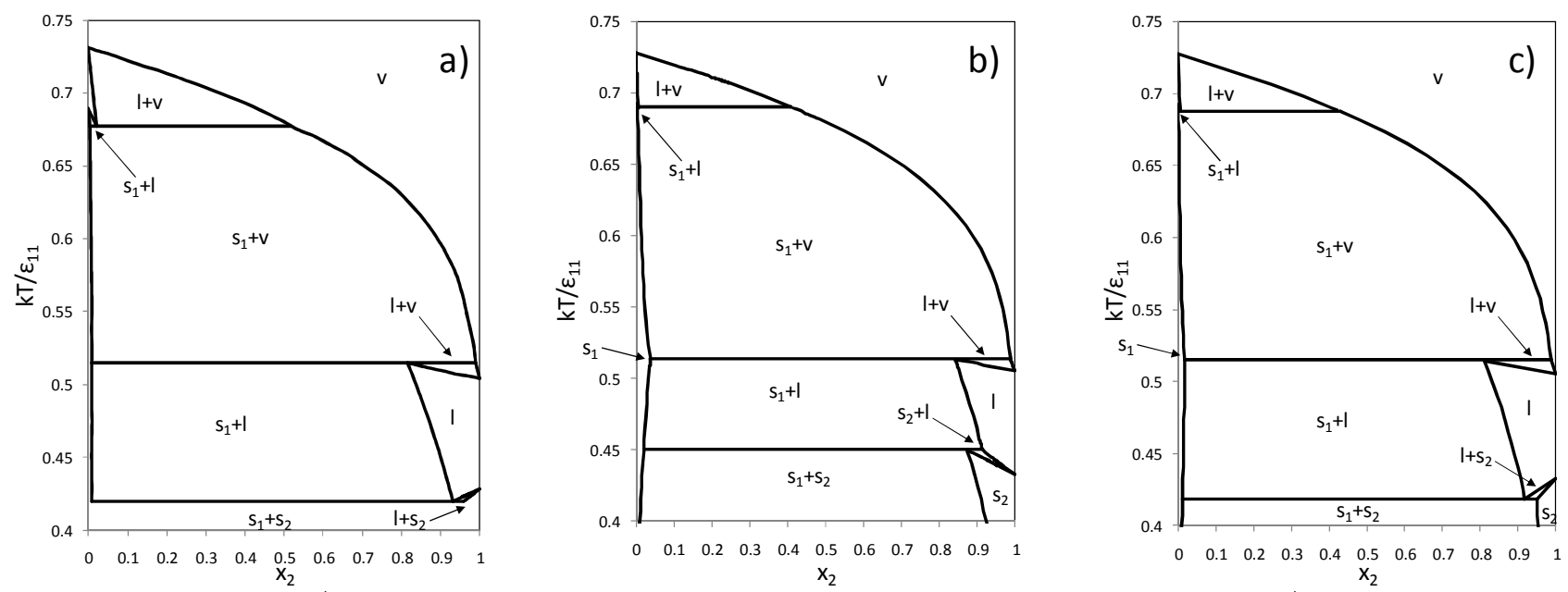

Figure 11. $T^{*}-\mathrm{x}_{2}$ phase diagrams for LJ binary mixtures with $\varepsilon_{11} / \varepsilon_{22}=1.6$ and $\sigma_{11} / \sigma_{22}=0.85$ at $P^{*}=0.002$.

(a) MC simulation diagram from [3]; (b) LJ SLV-EoS with null BIPs; (c) LJ SLV-EoS with regressed BIPs.

Table 2. Binary interaction parameters of the LJ SLV-EoS fort the studied mixtures.

\begin{tabular}{cccccc}
\hline$\varepsilon_{11} / \varepsilon_{22}$ & $\sigma_{11} / \sigma_{22}$ & $k_{12}=k_{21}$ & $l_{12}=l_{21}$ & $m_{12}=m_{21}$ & $n_{12}=n_{21}$ \\
\hline 0.625 & 0.95 & $-8.8 \times 10^{-4}$ & $1.13 \times 10^{-1}$ & $4.47 \times 10^{-2}$ & $9.96 \times 10^{-2}$ \\
1 & 0.95 & 0 & $1.5 \times 10^{-2}$ & 0 & $1.5 \times 10^{-2}$ \\
1.6 & 0.95 & $-2.32 \times 10^{-3}$ & $6.21 \times 10^{-3}$ & $-8.65 \times 10^{-3}$ & $-3.04 \times 10^{-3}$ \\
0.625 & 0.9 & $6.79 \times 10^{-3}$ & $9.99 \times 10^{-2}$ & $-1.35 \times 10^{-2}$ & $8.98 \times 10^{-2}$ \\
1 & 0.9 & $1.47 \times 10^{-3}$ & $7.69 \times 10^{-2}$ & $1.28 \times 10^{-3}$ & $7.33 \times 10^{-2}$ \\
1.6 & 0.9 & $1.44 \times 10^{-3}$ & $2.38 \times 10^{-2}$ & $-1.21 \times 10^{-2}$ & $2.18 \times 10^{-2}$ \\
0.625 & 0.85 & $1.51 \times 10^{-2}$ & $1.44 \times 10^{-1}$ & $-7.52 \times 10^{-2}$ & $1.55 \times 10^{-1}$ \\
1 & 0.85 & $-5.50 \times 10^{-2}$ & $1.32 \times 10^{-1}$ & $-1.07 \times 10^{-1}$ & $1.24 \times 10^{-1}$ \\
1.6 & 0.85 & $-4.89 \times 10^{-2}$ & $1.58 \times 10^{-1}$ & $-3.89 \times 10^{-2}$ & $1.38 \times 10^{-1}$ \\
\hline
\end{tabular}

\section{Conclusion}

The SLV-EoS has been compared with molecular simulation results for LJ pure fluids and binary mixtures. The representation of pure fluids phase diagram is satisfactory for VLE, and SVE. Less accurate representation is obtained for SLE at temperatures lower than the critical temperature. Concerning binary LJ mixtures, the LJ SLV-EoS with null binary interaction parameters produces only solid solution type phase diagrams for the whole range of $\sigma_{11} / \sigma_{22}$ and $\varepsilon_{11} / \varepsilon_{22}$. This is because the solid-solid miscibility is always overestimated by the LJ SLV-EoS. BIPs were regressed in order to represent qualitatively the MC simulation phase diagrams. BIPs allow representing the three types of phase diagrams: solid solution, solid azeotrope and simple eutectic. As future work, binary interaction parameters in the SLV-EoS can be obtained as function of $\varepsilon_{11} / \varepsilon_{22}$ and $\sigma_{11} / \sigma_{22}$, allowing predicting the phase behavior of other LJ mixtures (not used in the parameters regression procedure) only knowing the $\mathrm{LJ}$ parameters of the pure components. At the same time, phase diagrams for mixtures of real (spherical-like) fluids can be qualitatively predicted once the LJ parameters for the fluids are known.

\section{References}

1. A. Yokozeki, Int J Thermophys. 24589 (2003)

2. M.R. Hitchcock, C.K. Hall, J. Chem. Phys. 110 11433 (1999)

3. M.H. Lamm, C.K. Hall, AIChE J. 471664 (2001)

4. M.H. Lamm, C.K. Hall, Fluid Phase Equibria 18237 (2001)

5. M.H. Lamm, C.K. Hall, Fluid Phase Equibria 194 197 (2002)

6. M. Matsuoka, Bunri Gijutsu 7245 (1977)

7. J. Gmehling, B Kolbe, Thermodynamik, $2^{\text {nd }}$ ed., VCH-Verlag, Weinheim (1992)

8. A. Lotfi, J. Vrabec, J. Fischer, Molecular Physics 76 1319 (1992)

9. M.A. Barroso, A.L. Ferreira, J. Chem. Phys. 116 7145 (2002) 